\title{
Single- and Multi-User IPs' Users: Identical Seeking but Different Clicking
}

\author{
Nikolai Buzikashvili \\ Institute of System Analysis, Russian Academy of Sciences, Moscow, Russia
}

\begin{abstract}
In the study, users of the a Web search engine are partitioned into different classes according a number of users submitted queries from the same IP address. Seeking and clicking behavior of these "IP classes" is investigated. It is shown that seeking behavior of users from different classes is practically identical in all characteristics except their click behavior. It is shown that real-life environmental stressors do not affect query formulation or session execution, shift slightly moving across the retrieved results and shift greatly click behavior.
\end{abstract}

\section{Introduction}

A lot of studies look at click behavior and its uniform models. Some studies research individual and task differences in click behavior of different users ([3], [4], [5], [9]). Web log analysis reveals different manners of real-life search and clicking, but the effect of individual/task differences and environmental factors on these differences is not clear from this analysis.

Psychological studies (e.g., [8]) investigate the effects of work environment on the worker's performance. Several papers investigate web search behavior depending on different environmental conditions (e.g., [6], [7]).

In this web log-based study, we indirectly reveal the influence of real-life environmental factors and compare search behavior of users who are mainly "stressed-out office workers" or mainly "free homebodies". We can assume that when an office worker search is aimed at his office responsibilities rather than personal goals he must perform other functions and may be overloaded by them. We presume that his searching behavior under office stressors differs from his behavior in a relaxed atmosphere. We also presume that the shift of search behavior under varying environmental conditions reflects not so much individual manners but a common environmental dependency.

We investigate not the influence of individual features such as "advanced user" or "newbie" but the influence of the real-life environmental factors on search behavior of [the same] searchers.

The opportunity of this long-scale real-life comparison is provided by the "IP classes" consideration. The IP classes of users are defined in the following way: the IP-N class includes all users who share the same IP address with (N-1) other users. There are two reasons why a search engine detects different users operating from the same IP: (1) different browsers on the same PC, (2) different PCs sharing the same proxy server. As a result, IP-1 corresponds to individual users, IP-2 may correspond either to two users who use different browsers on the same PC or to a proxy presenting two PCs, and the IP-3+ classes mainly correspond to users sharing the same proxy.

A fraction of (from-)home users among the IP-1 users appears to be bigger than among the IP-3+ (i.e. proxy) users. Of course, there is no strong relation between home/office and IP-1/IP-3+. Some of the IP-99 users may be cliff dwellers whilst an IP-1 user may be a small enterprise worker. However, the rate of cookies created on weekend (see Table 1) is a good indirect indicator of fractions of home/office users, and this rate in the IP-1 class is bigger than in IP-3+ classes.

Also, while there is no a strong relation between an office and noisy environment, and home and relaxed atmosphere (the counterexamples are obvious just as some fishes can fly and some birds cannot fly), we can assume that on average there is more stressed-out search behavior at work and more free behavior at home [8].

In the study, we consider the characteristics of search behavior of different IP classes and discover that all of them are very similar except that of the click activity. Frequencies of clicks per query or per first page of the retrieved results are about $30 \%$ bigger for the IP-1 users than for the IP-4+ users. This difference can be explained neither by the topical difference between "home" and "office" queries nor by different ways of moving across the pages of the retrieved results. Thus, the study discovers a strong non-individual difference in click behavior between "free home" users and "busy office" ones. However, we do not know whether free home squirrels/ birds visit useless sites or busy office workers ignore the necessary ones.

Structure of the paper. The notion of IP class is introduced in Chapter 2. The research questions and assumptions are formulated in Chapter 3. Chapter 4 describes the source dataset used in the study and 
resulting IP-classes' datasets. Terms and a method of the study are presented in Chapter 5. General characteristics of seeking and clicking of IP classes are investigated in Chapter 6, and a great difference in click behavior of IP classes is reveled. Since this difference may be a result of (1) different frequency of different search topics in queries of different IP classes and (2) different topics may induce different seeking and clicking behavior, in Chapter 7 we compare characteristics of the same topical search among IP classes. The same inter-class difference is observed in the same topics search. Next, one can expect the difference in different (more complex and more simple) types of query modifications. Chapter 8 devoted to a detail study of query modification by different classes. Finally, in Chapter 10, clicking and viewing in different IP classes are investigated is in more detail as a probabilistic process.

\section{IP classes}

Web users may share the same IP address either if they use different browsers on the same PC or they operate via the same proxy server. If $3+$ users have the same IP address, they probably use a common proxy rather than $3+$ browsers on the same PC.

IP group. An IP group is a set of users who submit queries from the same IP address.

IP class. If an IP group includes $N$ users, the users are attributed to the IPN class. We also refer to queries, sessions, clicks, etc. of users belonging to the IPN class as IPN class queries, sessions, etc

\section{Research questions and assumptions}

\subsection{Research questions}

Are real-life working conditions stressful enough to change user search behavior? In particular, are the following features different in (differently stressed) IP classes:

\section{A query level:}

terms per a query,

clicks per a query,

viewed pages (screens) of the retrieved results per a query,

clicks per viewed pages,

clicks on the first page of the results.

\section{A search session level:}

task sessions per a temporal session,

queries per a task session,

click behavior in different types of task sessions: single-query sessions, sessions with linear query modification, sessions with branching query modification clicks in query narrowing and broadening modifications.

Besides, if the clicks and viewed pages of the retrieved results are actually different in the "office" IP-3, 4+ classes from the "home" IP-1 class then we should test two hypotheses "once started they will continue": if a user from the "office class" made the first click then he will continue clicking similarly to a user from the "home" IP-1 class; if a user from the "office class" moved to the next page of the retrieved results he will continue moving through pages just as the user from the "home" class.

In the study of the environment influence on search, we do not focus on "a user as a sequence of his operations during a long observation period". "User" is an irrelevant unit, not in the sense that different people can operate from the same UID but meaning that the same person may be busy or free. Even office work has breaks and pauses and even stay-at-homers may be unexpectedly busy. Whereas user is an irrelevant unit, query is a minimum unit and task session (and even temporal session) is a convenient unit representing stable ("busy" or "free") behavior.

\subsection{Assumptions}

We assume that:

a fraction of home users in the IP-1 class is significantly bigger than in the IP-4+ class, and a fraction of home users in the IP $K$ class is not smaller than in the IP- $(K+1)$ class, home users are less stressed-out by their environment.

At weekends and on holiday days the degree of activity of office workers decreases more than of home users, and it can be assumed that the rate of office cookies created at weekends is less than that of home users' cookies. The data in Table 1 support the first assumption: the rate of cookies created on Sunday decreases over the IP classes, and is twice as little for the IP-4+ class than for the IP-1 class.

Table 1. Fractions of cookies created on Sunday

\begin{tabular}{c|c|c|c}
\hline IP-1 & IP-2 & IP-3 & IP-4+ \\
\hline $8.5 \%$ & $8.5 \%$ & $7.5 \%$ & $4.4 \%$ \\
\hline
\end{tabular}

\section{Datasets and preprocessing}

We use a complete one-day dataset (March 20, 2007) drawn from the logs of the Yandex search engine. The dataset combines three logs (a query log, a $\log$ of the results and a click-through $\log$ ) and reports queries, retrieved results and clicks on them.

The source dataset was "cleaned up": users who submitted more than 40 unique queries per 1-hour are eliminated as "robots". To segment a logged time 
series of transactions into temporal sessions a $30 \mathrm{~min}$ cut-off was used.

The users in the dataset are represented by unique UIDs where UID is a concatenation of IP-part $=$ $<$ hash (IP address) $>$ and 10-digit UIDCreationTimepart $=<$ time_of_cookie_creation $>$, which allows us to detect all users who share the same IP address (the later Yandex public datasets formats do not provide this useful feature).

According to common IP-parts of UIDs the cleaned-up dataset was separated into four datasets: IP-1 (contains logged information on UIDs with unique IP-parts), IP-2 (contains information on UIDs belonging to IP-2 class), IP-3 (UIDs belonging to IP3 class) and IP-4+. Thus, IP-1, ..., IP-4+ datasets present different IP classes.

Table 2 gives statistics on these IP-classes datasets.

Table 2. General characteristics of IP class datasets

\begin{tabular}{c|c|c|c|c}
\hline & IP-1 & IP-2 & IP-3 & IP-4+ \\
\hline Users & 656557 & 98790 & 35022 & 27434 \\
\hline $\begin{array}{c}\text { Temporal } \\
\text { sessions }\end{array}$ & 1105496 & 163021 & 56064 & 43464 \\
\hline $\begin{array}{c}\text { Queries unique } \\
\text { in a temporal } \\
\text { session }\end{array}$ & 2463767 & 367193 & 125074 & 95815 \\
\hline Task sessions & 1632796 & 241374 & 82264 & 63374 \\
\hline
\end{tabular}

\section{Terms and method}

In the paper, we use the notions of:

- a query skeleton that include only those query terms that are nouns, names, acronyms and unknowns which may be attributed to these parts of speech, a skeleton includes neither features (adjectives, ) nor actions (verbs, adverbs),

- a query narrowing here denotes an expansion a query by additional term(s), and a query broadening here denotes an exclusion of one or more terms from a query (e.g., when a user submits query $\langle$ cat $\rangle$ after submission of $\langle$ red cat $\rangle$ he narrows the initial query, a modification of $\langle$ cat toys $\rangle$ into $\langle$ toys $\rangle$ is a broadening, and a modification of $\langle$ cat toys $>$ into <cat food> is neither narrowing nor broadening),

- a temporal session as a sequence of the user's transactions with the search engine cut from previous and successive sessions by a 30 min time gap;

- a task session as all queries of a task session technically defined as a connected component of the similarity graph of queries submitted during a time session. To extract task sessions (a) a matrix of termbased pairwise similarity of all unique queries of the current temporal session is filled (two queries are defined as similar if they contain common skeleton terms), (b) a transitive closure of this similarity relation is made [1]. The method [1] covers misprints in queries.

\section{Features on all queries}

\subsection{Query level features}

The general query-level features of IP classes are reported in Table 3.

Table 3. Features of IP classes

\begin{tabular}{c|c|c|c|c}
\hline & IP-1 & IP-2 & IP-3 & IP-4+ \\
\hline terms in a query & 3.02 & 3.01 & 3.01 & 2.98 \\
\hline $\begin{array}{c}\text { terms in a query } \\
\text { skeleton }\end{array}$ & 2.18 & 2.18 & 2.19 & 2.18 \\
\hline $\begin{array}{c}\text { viewed pages per } \\
\text { query }\end{array}$ & 1.58 & 1.57 & 1.54 & 1.50 \\
\hline clicks per query & $\mathbf{1 . 6 4}$ & $\mathbf{1 . 5 0}$ & $\mathbf{1 . 3 5}$ & $\mathbf{1 . 2 6}$ \\
\hline $\begin{array}{c}\text { clicks per viewed } \\
\text { page }\end{array}$ & $\mathbf{1 . 0 4}$ & $\mathbf{0 . 9 6}$ & $\mathbf{0 . 8 8}$ & $\mathbf{0 . 8 4}$ \\
\hline $\begin{array}{c}\text { clicks on the first } \\
\text { page }\end{array}$ & $\mathbf{1 . 2 5}$ & $\mathbf{1 . 1 5}$ & $\mathbf{1 . 0 5}$ & $\mathbf{0 . 9 9}$ \\
\hline
\end{tabular}

Users from all IP classes formulate queries identically (see "terms in a query" and "terms in a query skeleton"). The number of moves between pages of the retrieved results only slightly decreases over IP classes.

On the contrary, the click-based characteristics demonstrate big differences among classes. A number of clicks per a submitted query, a number of clicks per a viewed page of the retrieved results and a number of clicks on the first page of the retrieved results monotonously decreases over IP classes.

\subsection{Session level features}

The general session-level features of IP classes are reported in Table 4 . While a number of temporal sessions during a day steadily decrease over IP classes from 1.68 at IP-1 to 1.58 at IP-4+, the average numbers of task sessions in a temporal session are similar, and the average numbers of queries in a task session are practically identical. Thus, users in the IP-3+ classes start session less often but once started they behave similarly to IP-1 users.

Table 4. Features of IP classes. Session level

\begin{tabular}{l|r|c|c|c}
\hline & IP1 & IP2 & IP3 & IP4+ \\
\hline temporal sessions per user & 1.68 & 1.65 & 1.60 & 1.58 \\
\hline task sessions per temporal one & 1.48 & 1.48 & 1.47 & 1.46 \\
\hline queries per task session & 1.51 & 1.52 & 1.52 & 1.51 \\
\hline
\end{tabular}

Fractions of broadening and narrowing query reformulations in task sessions are the same across all IP classes.

\section{Features in topic dimensions}

Different search topics lead to different search behavior. Topics may be differently presented in 
queries of different IP classes and between-class differences may be the result of the differences in the topic occurrence. To check a uniformity of the discovered dependencies we investigate search behavior on three topics.

We consider two topics - "Travel" and "Education". We choose 20 topic-specific terms for the Travel topic and 10 topic-specific terms for Education. If a query contains a topic term, this query is considered as the "topical" one. If a task session contains a topical query than the session is considered as a topical session. Table 5 shows the number of topical queries, topical sessions and queries belonging to the sessions in IP classes.

Table 5. Topical queries and queries in topical task sessions

\begin{tabular}{|c|c|c|c|c|}
\hline & IP-1 & IP-2 & IP-3 & IP-4+ \\
\hline \multicolumn{5}{|l|}{ Topical queries } \\
\hline Travel queries & 45689 & 6246 & 2103 & 1556 \\
\hline Education queries & 52871 & 7548 & 2434 & 1766 \\
\hline \multicolumn{5}{|c|}{ Queries in topical sessions } \\
\hline $\begin{array}{l}\text { Queries in travel } \\
\text { sessions }\end{array}$ & 68412 & 9399 & 3187 & 2416 \\
\hline $\begin{array}{l}\text { Queries in education } \\
\text { sessions }\end{array}$ & 79499 & 11395 & 3671 & 2624 \\
\hline
\end{tabular}

The results in Table 6 show the same dependencies that were observed on all queries: (1) similar values of queries per task session, (2) a slightly decreasing number of viewed pages and (3) a sharply decreased number of clicks over IP classes.

Table 6. Queries in all and topical task sessions

\begin{tabular}{l|l|l|l|l}
\hline & IP-1 & IP-2 & IP-3 & IP4+ \\
\hline Queries per task session in: & \multicolumn{5}{l}{} \\
\hline All sessions & 1.51 & 1.52 & 1.52 & 1.51 \\
\hline Travel sessions & 2.23 & 2.25 & 2.25 & 2.36 \\
\hline Education sessions & 2.45 & 2.45 & 2.45 & 2.37 \\
\hline Clicks per query in: & 1.64 & 1.50 & 1.35 & 1.26 \\
\hline All sessions & 1.77 & 1.65 & 1.62 & 1.35 \\
\hline Travel sessions & 1.95 & 1.72 & 1.56 & 1.61 \\
\hline Education sessions & 1.58 & 1.57 & 1.54 & 1.50 \\
\hline Viewed pages per query in: \\
\hline All sessions & 1.59 & 1.57 & 1.56 & 1.57 \\
\hline Travel sessions & 1.74 & 1.76 & 1.66 & 1.68 \\
\hline Education sessions & 1.04 & 0.96 & 0.88 & 0.84 \\
\hline Clicks per viewed pages in: \\
\hline All sessions & 1.12 & 1.05 & 1.03 & 0.86 \\
\hline Travel sessions & 1.08 & 0.98 & 0.91 & 0.96 \\
\hline Education sessions & 1.25 & 1.15 & 1.05 & 0.99 \\
\hline Clicks on the first page in: \\
\hline All sessions & 1.39 & 1.29 & 1.27 & 1.09 \\
\hline Travel sessions & 1.40 & 1.26 & 1.18 & 1.15 \\
\hline Education sessions
\end{tabular}

\section{Different types of query reformulation}

We observed great difference between IP classes in clicking. One can expect the difference in different (more expensive and more easy) types of query modifications. To check this opportunity we investigate in this chapter difference in different types and characteristics of query modifications.

\subsection{A model of query reformulation process}

According to [2] a task session may include several branches and a complex search task may be decomposed into chains which merge in the final step. We suppose that:

(1) a query may depend on non-direct predecessor: $Q_{T}=f\left(Q_{S<T}\right)$ instead of $Q_{T}=f\left(Q_{S=T-1}\right)$. As a result, several queries may depend on the same query (a branching search: queries $Q_{T 1}=f\left(Q_{S}\right), \ldots$ $Q_{T N}=f\left(Q_{S}\right)$ depend on the same query $\left.Q_{S}\right)$. A model doesn't limit a number of chains $\left\{Q_{S}=>Q_{T_{1}}[=>\ldots]\right\}$ which have the same root $Q_{S}$

(2) a query may be a "combination" of a pair of earlier submitted queries $Q_{T}=f\left(Q_{S 1<T}, Q_{S 2<T}\right) \quad$ (a merging search) rather than a modification of a single query. A model restricts a number of determining predecessor by a pair as a maximum. This restriction is motivated by neurophysiological mechanisms of human information processing according to which parallel execution of more than two tasks (finally merging query modification chains) is practically impossible.

Interpretations of the same series of user queries vary depending on the query dependencies taken into account. Table 7 shows differences between linear and non-linear interpretations of two real life time serieses of user queries.

Table 7. Observed sequence of user queries and its interpretations

\begin{tabular}{|c|c|c|}
\hline $\begin{array}{l}\text { Observed } \\
\text { sequence }\end{array}$ & $\begin{array}{c}\text { Linear } \\
\text { interpretation }\end{array}$ & $\begin{array}{c}\text { Non-linear } \\
\text { interpretation }\end{array}$ \\
\hline $\begin{array}{l}Q_{1}=\text { Chinese } \\
\text { nosh cook } \\
\text { wanted } \\
Q_{2}=\text { Chinese } \\
\text { nosh } \\
Q_{3}=\text { Chinese coo } \\
\text { wanted }\end{array}$ & $\begin{array}{l}Q_{1} \stackrel{\text { broad }}{\longrightarrow} Q_{2} \text { vary } \\
Q_{1} \stackrel{\text { broad }}{\longrightarrow} \mathrm{Q}_{2}\end{array}$ & \\
\hline $\begin{array}{l}Q_{1}=\text { crown buy } \\
Q_{2}=\text { toyota mosco } \\
Q_{3}=\text { toyota crown } \\
\text { moscow }\end{array}$ & $\mathrm{Q}_{2}$ vary & \\
\hline
\end{tabular}

However, are the non-linearity assumptions (a) psychologically grounded and (b) technically supported by search engine interfaces? A branching search is not a surprising manner for any living being. A human searching on the Web comes up against a common situation provoking a branching search: when an initial query perfectly expresses an 
information need but retrieves unsatisfactory results, a user modifies the query; if the results of the modified query are also unsatisfactory, a user refines the initial query rather than the current one. A merging search is poorly supported by a human memory and is not supported by search environments. However, interviewing of ordinal searchers shows that both manners are consciously applied when a "direct search" gives unsatisfactory results. Besides, we can expect a lot of "unconscious" branching modifications of an initial query perfectly presenting an information need but retrieving poor results.

We use term-based query-on-queries dependencies as representatives of a query modification process. If there is a set of queries which may be determinants of a query (i.e. precede a query and have a non-zero influence on it according to a certain dependency measures), a query considered as modification of the recent among strongest determinants. All dependencies are detected for queries submitted during a short-time period (a time session). To determine dependency of a query only its first occurrence in a time session is used, and a query may depend on queries submitted before its first occurrence. The strongest dependency of a query is selected according to certain decision rules.

Let dependencies between queries be presented by a graph of a logical structure of a search, in which each query be presented by a unique node regardless of a number of occurrences of the query in a session. The connected components of this graph correspond to "task sessions". The same logical structure may present different real sequences of user's transactions. Different tasks or different branches of the same task may alternate in execution and even linear tasks may be executed non-linearly: one task is broken by another task and restored again (occasional non-linearity).

Let distinct queries submitted by the same user during a time session be presented by nodes and dependencies (the strongest dependencies according to a certain dependency measure, which will be set in a form of 2-step decision rule) between queries be presented by arcs. We will refer to this directed acyclic graph as a query modification graph. According to (2) a maximum indegree is equal to 2; according to (1) outdegrees are not limited. If there are two paths from the node $\mathrm{R}$ to the node $\mathrm{M}$ crossing only in $\mathrm{R}$ and $\mathrm{M}$ we speak about re-merging (merging of earlier branched chains) and call $\mathrm{H}$ a root of re-merging.

Table 8 shows synthetic examples of basic (branching, merging) and derivative non-linearities (re-merging and occasional non-linearity of execution) in a search process and its logical structure.
Table 8. Non-linearities in logical structure and in observed sequence of execution

\begin{tabular}{|l|l|l|}
\hline $\begin{array}{c}\text { Sequence of } \\
\text { execution }\end{array}$ & $\begin{array}{c}\text { Dependencies } \\
\text { in logical } \\
\text { structure }\end{array}$ & $\begin{array}{c}\text { Dependencies } \\
\text { in sequence of } \\
\text { execution }\end{array}$ \\
\hline $\begin{array}{l}\text { Occasional } \\
\text { (non-linear execution of linearly dependent queries) }\end{array}$ \\
$\begin{array}{l}\text { kitten food }-1 \\
\text { cat food }-2\end{array}$ \\
$\begin{array}{l}\text { query } \\
\text { query modificat-4 } \\
\text { hills cat diet }-5\end{array}$
\end{tabular}

An applicable non-linear model (1)-(2) presents a general framework to describe a query modification process. There are a lot of technical variants of the model implementation. Here, we use several rules of structuring query modification process based on lexical dependency measures. Different kinds of dependency measures are sound and the best way to detect and to analyze a non-linear search is to use a family of decision rules, each of which may operate with different measures (e.g., combine them).

\subsection{Features of linear and branching query reformulations}

The most significant types are linear and branching query modification. Here we use Jaccard part-of-speech-based similarity metric to detect interquery dependencies in a task session. For example, branching search will be detected in the sequence of

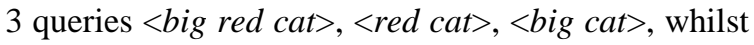
a linear modification is detected in the sequence $<$ red cat $\rangle,\langle$ cats food $\rangle$, <cats veterinary $\rangle$..

Table 9 shows practically identical rates of 3 types of temporal sessions among temporal sessions of IP classes. 
Table 9. Types of temporal sessions

\begin{tabular}{l|c|c|c|c}
\hline & IP-1 & IP-2 & IP-3 & IP-4+ \\
\hline Total & 1109481 & 163577 & 56225 & 43569 \\
\hline $\begin{array}{l}\text { Single-query } \\
\text { temporal } \\
\text { sessions }\end{array}$ & $52.14 \%$ & $51.35 \%$ & $51.84 \%$ & $51.73 \%$ \\
\hline $\begin{array}{l}\text { Linear } \\
\text { temporal } \\
\text { sessions }\end{array}$ & $41.27 \%$ & $41.95 \%$ & $41.85 \%$ & $42.09 \%$ \\
\hline $\begin{array}{l}\text { Non-linear } \\
\text { temporal } \\
\text { sessions }\end{array}$ & $6.59 \%$ & $6.70 \%$ & $6.31 \%$ & $6.18 \%$ \\
\hline
\end{tabular}

Table 10 shows an average number of clicks per components of single-query, linear and branching task sessions. We see two sorts of differences: (1) the difference between IP-classes and (2) replicated in all IP classes differences between different structures of search and between query position in the same structure.

Table 10. Clicks per chain of query reformulations in three structures of query modifications

\begin{tabular}{|c|c|c|c|c|}
\hline Chain length & IP-1 & IP-2 & IP-3 & IP4+ \\
\hline Single-query task session & 1.63 & 1.53 & 1.40 & 1.40 \\
\hline \multicolumn{5}{|c|}{ Linear chain of query modification } \\
\hline 2-query & 3.38 & 3.08 & 2.70 & 2.56 \\
\hline 3-query & 4.62 & 4.11 & 3.72 & 3.54 \\
\hline \multicolumn{5}{|c|}{ Branches (including a root query) } \\
\hline \multicolumn{5}{|l|}{ First branch } \\
\hline 2-query & 3.14 & 2.84 & 2.74 & 2.22 \\
\hline 3-query & 4.38 & 3.81 & 3.78 & 3.66 \\
\hline \multicolumn{5}{|l|}{ Last branch } \\
\hline 2-query & 3.74 & 3.34 & 3.08 & 2.52 \\
\hline 3-query & 4.92 & 4.41 & 4.26 & 3.45 \\
\hline
\end{tabular}

Clicks dependency on a query position in a search structure is near identical among IP classes, but belonging to IP class shifts click values. For example, Figures 1 and 2 show an average number of clicks in positions of 2- and 3-query linear chains of linear query modification. These IP classes' plots look like results of a parallel shift of the same plot.

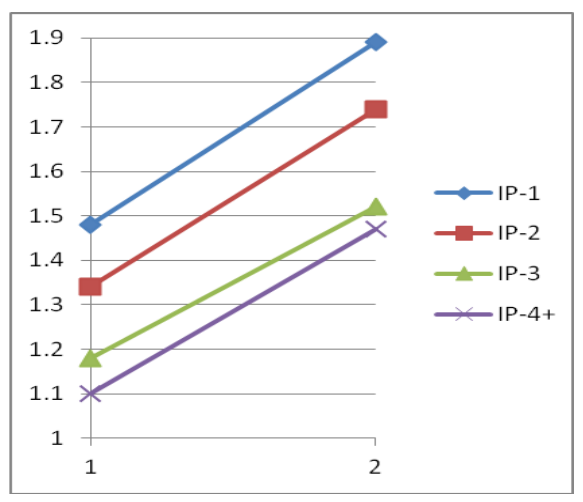

Figure 1. Average number of clicks in positions of 2-query linear chains

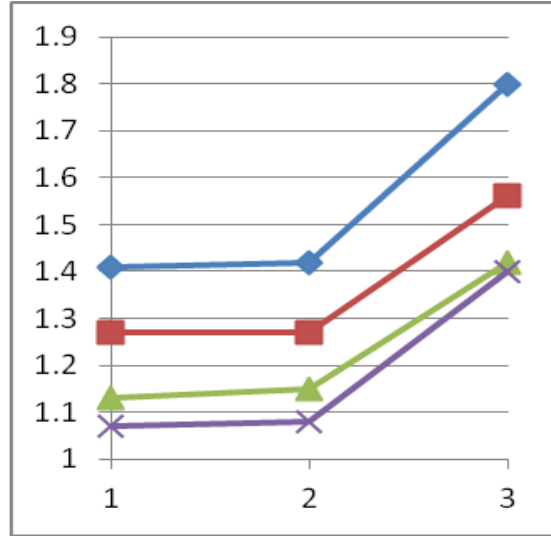

Figure 2. Average number of clicks in positions of 3-query linear chains

Clicks dependency on a query position in a search structure is near identical among IP classes, but belonging to IP class shifts click values. For example, Figures 1.1 and 1.2 show an average number of clicks in positions of 2- and 3-query linear chains of linear query modification. These IP classes' plots look like results of a parallel shift of the same plot.

Query narrowing and broadening in linear and branching query modifications. Table 11 presents IP classes in terms of queries broadening and narrowing in linear and branching query reformulations (a query broadening denotes an exclusion of one or more terms from the initial query and a query narrowing here denotes an expansion a query by additional terms).

Table 11. Query narrowing and broadening in linear and branching query modifications

\begin{tabular}{r|r|c|c|c}
\hline & IP1 & IP2 & IP3 & IP4+ \\
\hline broadening reformulation: & & & & \\
in linear reformulations & $11.4 \% 11.4 \%$ & $11.4 \%$ & $11.4 \%$ \\
in branching reformulations & $23.7 \% 23.6 \%$ & $24.1 \%$ & $24 \%$ \\
\hline narrowing reformulation: & & & & \\
in linear reformulations & $38.3 \% 38.2 \%$ & $38.5 \%$ & $38.3 \%$ \\
in branching reformulations & $14.2 \% 14.2 \%$ & $15.4 \%$ & $13.6 \%$ \\
\hline
\end{tabular}

We see a great difference between narrowings and broadenings in both kinds of query reformulations. But we see no difference between IP classes, rates of narrowing/broadening in both types of modifications are practically identical in all IP classes.

\section{Resume}

No noticeable difference in search behavior is observed between classes except the only, but great difference in clicks. Any click-based query- or session-level characteristic (clicks per a query, per viewed pages, per first viewed page, clicks in any type of query modification chains) sharply decrease over IP classes. 


\section{Clicks and viewed pages of the retrieved results}

\subsection{Differences between IP classes in clicks and viewed pages distributions}

Tables 3 and 6 present mean values of clicks per query. Let's consider clicking and moving in more detail. Distributions of clicks per query are reported in Table 12. Also we consider "clicks per the first page of the retrieved results" and "viewed pages per query" distributions.

We compare clicks per query distributions of the IP classes on $[L, 15+]$ intervals of clicks per query $(L=0, ., 14)$ by $\chi^{2}$ test. A log-scaled Figure 2 shows empirical and critical $\chi^{2}$ values. A sample curve in a point $L$ presents empirical $\chi^{2}$ value for the interval $[L, 15+]$, and a critical curve shows critical $\chi^{2}$ value at $p=0.05$ for degrees of freedom for $<4$ sets, $15-L$ set size $>$. Distributions on $[L, 15+]$ are similar beginning with $L=3$.

Table 12. Clicks per query distributions in IP classes

\begin{tabular}{c|r|r|r|r|r|r}
\hline & \multicolumn{1}{|c|}{$\mathbf{0}$} & \multicolumn{1}{|c|}{$\mathbf{1}$} & \multicolumn{1}{c|}{$\mathbf{2}$} & \multicolumn{1}{|c|}{$\mathbf{3}$} & \multicolumn{1}{|c}{} & \multicolumn{1}{|c}{$\mathbf{1 5 +}$} \\
\hline IP-1 & 1010108 & 650483 & 295077 & 172617 & $\ldots$ & 16671 \\
\hline IP-2 & 165656 & 91237 & 41113 & 23657 & $\ldots$ & 2195 \\
\hline IP-3 & 61506 & 29424 & 12994 & 7501 & $\ldots$ & 623 \\
\hline IP4+ & 49190 & 22161 & 9470 & 5297 & $\ldots$ & 420 \\
\hline
\end{tabular}

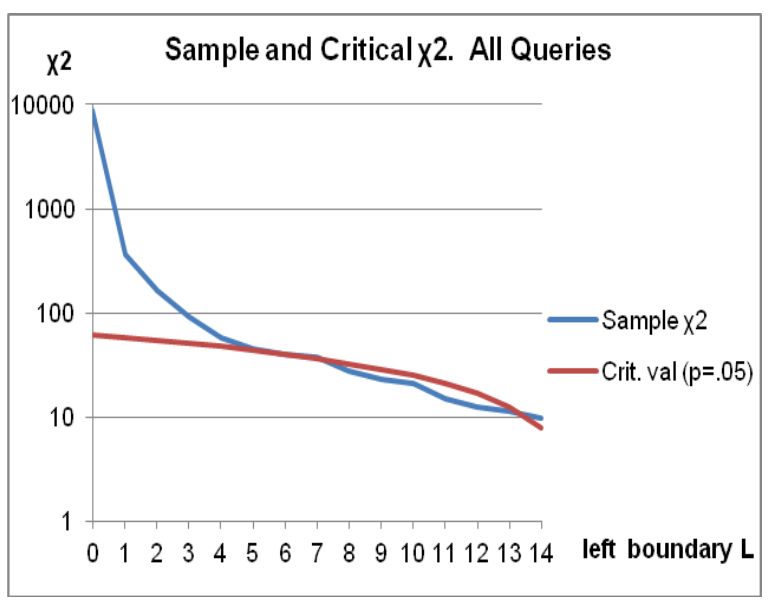

Figure 3. Sample $X^{2}([L, 15+])$ and critical $X 2(p=0.05)$ as functions of the left boundary $L=0, . ., 14$

As Figure 3 shows, click behaviors of IP classes strongly differ on the first clicks and are similar for subsequent clicks. Distributions of clicks on the first page of the results demonstrate just the same incoherence in the first clicks and coherence in the following clicks. At the same time, distributions of viewed pages are similar on all intervals $[L, 9+]$, where $L=1,,,, 8$.

\subsection{Description in terms of transition probabilities}

Let's return to the hypotheses "once started they will continue". As regards to click behavior, it means that empirical probabilities of the first click may differ between classes but probabilities of transition to the any following clicks are very similar.

Empirical transition probabilities of the next click on the results of the query and transition probabilities of the next click on the first page of the results are shown in Figures 3 and 4, where $p(c)$ is an empirical probability of the next click when $c$-1 clicks are made.

As Figures 4 and 5 show, probabilities for different IP classes greatly differ for the first click and are very similar for the further clicks. The trigger "once started" hypothesis is true for click behavior. A mainly stressful environment is not totally and continuously stressful and even a normally busy worker has some free time.

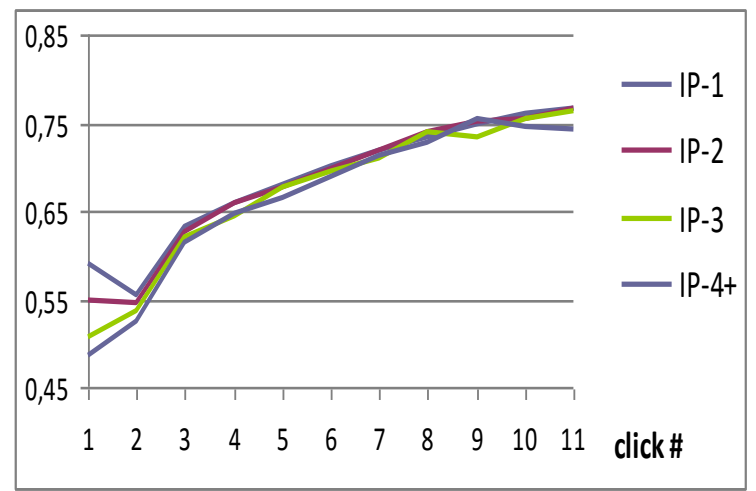

Figure 4. Probabilities of transition to click\# (a) on all viewed pages and (b) on the first page

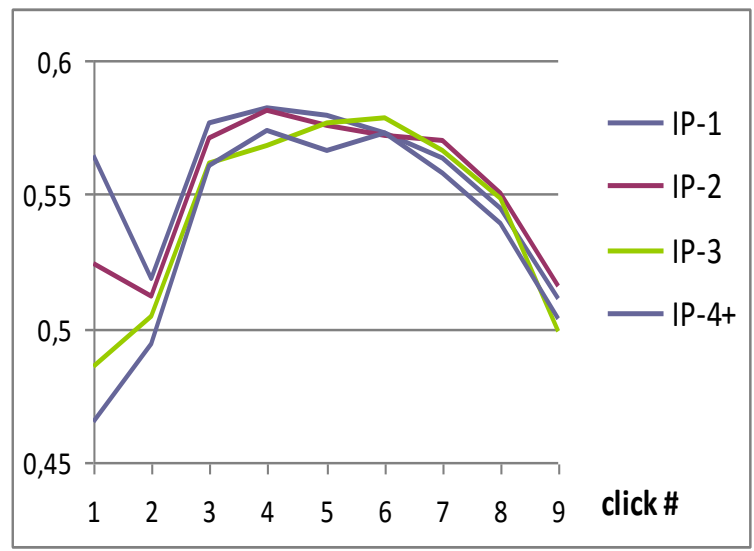

Figure 5. Probabilities of transition to click\# (a) on all viewed pages and (b) on the first page

Empirical transition probabilities of moving to the another page of the retrieved results are shown in Figure 6, where $p$ (move\#) is an empirical probability of move\#-th move to the another page (i.e. after viewing move\# retrieved pages). The probabilities 
are similar among all IP classes and moving across the retrieved results does not depend on the IP class. This is not a surprise since viewed pages per query distributions are similar among classes.

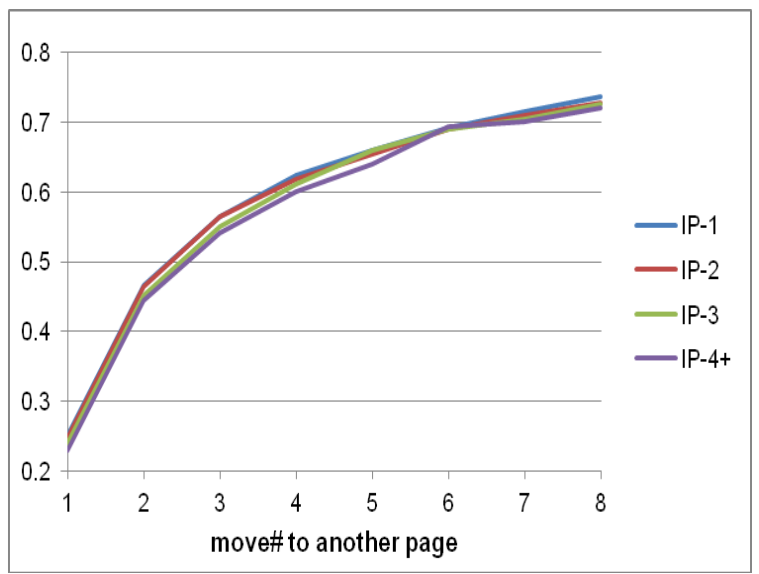

Figure 6. Probability of move\# to the another page of the retrieved results

\section{Conclusion}

The empirical study of IP classes gives answers to the questions about the influence of the environmental stressors on real-life search and click behavior of the Web user:

a query formulation/reformulation does not vary across IP classes and does not depend on real-life stressors,

the number of queries in task sessions, the number of task sessions in a temporal session and fractions of different types of task sessions do not vary across IP classes and do not depend on real-life stressors,

the number of the viewed pages of the retrieved results decreases slightly over IP classes,

the number of clicks (per query, per a viewed page of the retrieved results, on the first viewed page) and in all types of task sessions decreases over classes along with a fraction of free users.

The first click plays the trigger role: if a user in the "office IP class" started clicking he will continue clicking similarly to a "home" IP-1 class user (Figures 3 and 4). 0-click behavior frequent in IP-3+ classes seems to be a bit strange: "a busy user" has no time to visit the retrieved pages but has enough time to move through $2+$ pages of the retrieved results. Real-life environment stressors do not make searchers change query (re)formulation or significantly change the number of viewed pages but urge them to decrease the number of clicks.

The rates of IP classes have changed since 2007 and the fraction of IP-4+ class must have increased. However, it is not the IP classes which matter as such but the fact that since 2007 the share of home users (retired people, home workers), i.e. the share of "free users" with their non-stressed behavioral patterns has grown.

\section{References}

[1] Buzikashvili, N. (2006) "Automatic Task Detection in the Web Logs and Analysis of Multitasking", 9th Conferece ib Asian Digital Libraries, 2006, Kyoto, Japan, 2006, LNCS 4312, Springer-Verlag, 2006, pp. 131-140.

[2] Buzikashvili, N. (2009) "Non-Linear Query Reformulation Behavior," DIR-2009 (Enschede, Netherlands, 2009), Uni. Twente, 2009, pp. 42-50.

[3] Eickhoff, C., Teevan, J., White, R., and Dumais, S. (2014) "Lessons from the journey: a query log analysis of within-session learning", in Proceedings of WSDM'14, NY, USA ACM Press, ,2014, pp. 223-232.

[4] Jiang, J., He, D., and Allan, J. (2014) "Searching, Browsing, and Clicking in a Search Session: Changes in User Behavior by Task and Over Time", in Proceedings of 37th ACM SIGIR conf. on research and development in information retrieval, Gold Coast,Austalia,2014, ACM Press, pp. 607-616.

[5] Hu, B., Zhang, Y., Chen, W., Wang, G., and Yang, Q. (2011) "Characterizing search intent diversity into click models", in Proceedings of 20th World Wide Web Conference, Hyderabad, India. ACM Press, 2011, pp. 1726.

[6] Rieh. S.Y. (2004) On the Web at Home: "Information Seeking and Web Searching in the Home Environment", J. of the Association for Information Science and Technology, Vol. 55, no. 8, 2004, pp. 743-753.

[7] Sushmita, S., Joho, H., Lalmas, M., and Villa, R. (2010) "Factors affecting click-through behavior in aggregated search interfaces", in Proceedings of 19th Conf. Information and Knowledge Managemen, Toronto, 2010, ACM Press, 2010, pp. 519-528.

[8] Vischer, J.C. (2007) "The effects of the physical environment on job performance: towards a theoretical model of workspace stress", Stress and Health, vol. 23, no 3, 2007, Wiley, pp. 175-184.

[9] White, R., Dumais, S., and Teevan, J. (2009) "Characterizing the influence of domain expertise on web search behavior", in Proceedings of 2nd ACM Conf. on Web Search and Data Mining, Barcelona, Spain. ACM Press, 2009, pp. 132-141. 